\title{
Resting Heart Rate and Associated Factors in Patients with Chronic Heart Failure in Cameroon: A Cross-sectional Study in Sub-saharan Africa
}

\author{
Ba Hamadou ${ }^{1,2, *}$, Jérôme Boombhi, ${ }^{1,3}$, Audrey Joyce Foka ${ }^{4}$, Sylvie Ndongo Amougou ${ }^{1,5}$, \\ Liliane Mfeukeu-kuate ${ }^{1,2}$, Chris Nadège Nganou ${ }^{1,2}$, Aurel Tankeu ${ }^{4}$, Ahmadou Musa Jingi ${ }^{1}$, \\ Alain Menanga ${ }^{1,3}$, Kingue Samuel ${ }^{1,3}$ \\ ${ }^{1}$ Department of Medicine and Specialties, Faculty of Medicine and Biomedical Sciences, University of Yaoundé I, Yaounde, Cameroon \\ ${ }^{2}$ Cardiology Unit, Central Hospital of Yaounde, Yaounde, Cameroon \\ ${ }^{3}$ Cardiology Unit, Medicine B, General Hospital of Yaounde, Yaounde, Cameroon \\ ${ }^{4}$ Faculty of Medicine and Biomedical Sciences, University of Yaounde I, Yaounde, Cameroon \\ ${ }^{5}$ Cardiology Unit, University Teaching Hospital of Yaounde, Yaounde, Cameroon
}

Email address:

drhamadouba@yahoo.fr (B. Hamadou),jingiahmadoumusa@yahoo.co.uk (A. M. Jingi)

${ }^{*}$ Corresponding author

\section{To cite this article:}

Ba Hamadou, Jérôme Boombhi, Audrey Joyce Foka, Sylvie Ndongo Amougou, Liliane Mfeukeu-kuate, Chris Nadège Nganou, Aurel Tankeu, Ahmadou Musa Jingi, Alain Menanga, Kingue Samuel. Resting Heart Rate and Associated Factors in Patients with Chronic Heart Failure in Cameroon: A Cross-sectional Study in Sub-saharan Africa. Cardiology and Cardiovascular Research. Vol. 4, No. 1, 2020 , pp. 1-4. doi: $10.11648 /$ j.ccr.20200401.11

Received: December 25, 2019; Accepted: January 2, 2020; Published: January 17, 2020

\begin{abstract}
Introduction: Controlled heart rate has been associated with better prognosis in patients with chronic heart failure (CHF). Radial pulse palpation is a simple and useful method to evaluate heart rate control especially in limited resources setting such as sub-Saharan Africa. However, data on heart rate control in patients with heart failure are scarce in our context. Therefore, we sought to investigate heart rate control in patients followed for chronic heart failure in Cameroon. Methodology: We conducted a cross-sectional study from January to September 2017 in three specialized cardiology centers of Cameroon. We included patients with chronic heart failure, normal sinus rhythm without recent cardiac events. Resting heart rate was measured by pulse palpation after 05 minutes of rest, three (03) times over 60 seconds. An uncontrolled heart rate was defined as HR $\geq 70 \mathrm{bpm}$. Results: Overall, 213 patients with a mean age of $63 \pm 15$ years were included. About $64.8 \%$ of patients have a heart rate above recommended targets $(\geq 70 \mathrm{bpm})$. There was no significant association between age, gender, NYHA stage, cardiovascular risk factors or current therapies and heart rate control. Conclusion: Uncontrolled heart rate is frequent in patients with chronic heart failure in our context with more than half presenting a resting heart rate above currently recommended values.
\end{abstract}

Keywords: Heart Rate, Stable Chronic Heart Failure, Cameroon

\section{Introduction}

Heart failure affects about $2 \%$ of the general population and is the major complication of hypertension in sub-Saharan Africa. [1] Despite advances in medical and device therapy, the prognosis remains poor for patients with chronic heart failure (HF), with a mortality rate of $75 \%$ at 7 years of follow-up. Heart rate not only predicts the outcome but may also be a therapeutic target in patients with chronic heart failure. [2] An uncontrolled heart rate is known to have a detrimental effect on the occurrence of heart failure and is a well-established risk factor in patients with chronic heart failure [3-5]. Guidelines for the management of stable CHF recommend heart rate values below $70 \mathrm{bpm}$ to prevent myocardial ischemia and improve cardiac outcomes [6]. Indeed, it is now well established that patients with heart rate 
$\geq 70 \mathrm{bpm}$ have a higher risk of vascular outcomes compared with patients with heart rate less than 70 bpm [7-9]. Moreover, there is evidence that clinical benefits are tightly associated with the magnitude of heart rate reduction.

Despite the proven benefits of heart rate reduction, several observational studies suggest that this target is not systematically achieved in clinical practice. Our study explored heart rate control in the major heart failure centers throughout the country, Cameroon. We aimed to identify the proportion of patients with stable chronic heart failure attending heart failure clinics with a resting heart rate of $\geq 70$ bpm as well as to determine factors associated with poor heart rate control.

\section{Methods}

\subsection{Ethical Statement}

This study was approved by the Institutional and Research Ethical Committee of the faculty of medicine and biomedical sciences and we obtained administrative authorizations of the reference centers where the study was carried out. All participants provided written informed consent.

\subsection{Study Design and Sampling}

This was a cross-sectional study involving 213 patients from 03 major centers of heart failure management in Cameroon carried out from January 2017 to September 2017. These centers regularly follow patients with acute and chronic heart failure. We included all consenting adults in an out-patient setting with stable chronic heart failure and normal sinus rhythm. We excluded patients hospitalized for decompensated heart failure within the month prior to consultation and those presenting any conditions which could alter heart rate.

\subsection{Variables and Measurements}

Data were collected on demographic characteristics, risk factors and lifestyle, medical history, physical condition, and vital signs, records were made of heart failure therapies including the use and dosage of heart rate reducing agents. Resting heart rate was recorded by three separate radial pulse assessments and their mean was used for analysis. This was done in a quiet environment, with the patient in a sitting position, for 60 seconds.

\subsection{Statistical Analysis}

Statistical analyses were performed using IBM SPSS Statistics for Windows, version 23.0. Data are presented using descriptive statistics with numbers and percentages (\%) for categorical variables, and the mean \pm standard deviation for continuous variables. The chi-square test was used to study the association between two qualitative variables, while for the quantitative independent variables the association with heart rate was investigated by comparing the mean with a Student test. Binary logistic regression was used in multivariate analysis to determine factors associated with poor heart rate control. The statistical significance threshold was set at $\mathrm{p}<0.05$.

\section{Results}

Among the 310 patients enrolled, 213 patients met the inclusion criteria and were included in the final analysis. $64.8 \%(n=138)$ of participants had sub-optimal heart rate control, while $35.2 \%(n=75)$ were well controlled with heart rate less than 70 beats per minute (bpm). The characteristics of our patients are summarized in Table 1 .

Table 1. Characteristics of the patient with stabilized chronic heart failure.

\begin{tabular}{lll}
\hline Variable & Frequency $(\mathbf{N}=\mathbf{2 1 3})$ & Percentage (\%) \\
\hline Male & 82 & 38.5 \\
Female & 131 & 61.5 \\
Age (years) & & \\
$\geq 55$ & 160 & 75.1 \\
$<55$ & 53 & 24.9 \\
Diabetes & 50 & 23.5 \\
Hypertension & 145 & 68.1 \\
Smoking & 27 & 12.7 \\
Obesity & 63 & 29.6 \\
CKD & 18 & 8.5 \\
COPD / Asthma & 5 & 2.3 \\
Causes of HF & & \\
Ischemic & 29 & 13.6 \\
Non-ischemic & 184 & 86.4 \\
NYHA & & \\
1 & 26 & 12.2 \\
2 & 116 & 54.5 \\
3 & 71 & 33.3 \\
Beta blockers & 149 & 70 \\
Digoxine & 25 & 11.7 \\
Ivabradine & 1 & 0.5 \\
ACEi & 152 & 71.4 \\
ARB & 34 & 16 \\
Aldosterone antagonist & 98 & 46 \\
Diuretics & 173 & 81.2 \\
\hline
\end{tabular}

There were no differences among controlled and uncontrolled patients in the baseline characteristics namely age, sex, hypertension, diabetes, Left Ventricular Ejection Fraction, the etiology of systolic dysfunction, New York Heart Association (NYHA) functional class (Table 2).

Table 2. Factors associated with suboptimal heart rate control.

\begin{tabular}{llll}
\hline Variable & $\begin{array}{l}\text { Heart rate } \geq 70 \\
\text { bpm }(\mathbf{n}=\mathbf{1 3 8})\end{array}$ & $\begin{array}{l}\text { Heart rate }<\mathbf{7 0} \\
\text { bpm }(\mathbf{n}=\mathbf{7 5})\end{array}$ & $\boldsymbol{p}$ value \\
\hline Male (\%) & 39 & 37 & 0.912 \\
Female (\%) & 61 & 63 & \\
Age (years) & & & \\
$\geq 55$ & 75 & 25 & 0.376 \\
$<55$ & 25 & 75 & \\
Diabetes (\%) & 27 & 17 & 0.077 \\
Hypertension (\%) & 71 & 63 & 0.783 \\
Smoking (\%) & 12 & 13 & 0.832 \\
Obesity (\%) & 33 & 24 & 0.336 \\
CKD (\%) & 7 & 12 & 0.120 \\
COPD / Asthma (\%) & 2 & 3 & 0.999 \\
Causes of HF (\%) & & & 0.930 \\
Ischemic & 14 & 13 & \\
Non-ischemic & 86 & 87 & \\
\hline
\end{tabular}




\begin{tabular}{llll}
\hline Variable & $\begin{array}{l}\text { Heart rate } \geq 70 \\
\text { bpm }(\mathbf{n = 1 3 8})\end{array}$ & $\begin{array}{l}\text { Heart rate }<70 \\
\text { bpm }(\mathbf{n}=\mathbf{7 5})\end{array}$ & $\boldsymbol{p}$ value \\
\hline NYHA (\%) & & & \\
$\geq 3$ & 31 & 33 & 0.745 \\
$<3$ & 69 & 67 & \\
LVEF (\%) & & & \\
$\geq 35$ & 51 & 59 & 0.313 \\
$<35$ & 49 & 41 & \\
Betablockers (\%) & 67 & 75 & 0.269 \\
Digoxin (\%) & 12 & 11 & 0.720 \\
Ivabradine (\%) & 0 & 0 & 0 \\
ACEi (\%) & 70 & 73 & 0.639 \\
ARB (\%) & 19 & 11 & 0.090 \\
Aldosterone & 47 & 43 & 0.471 \\
antagonist (\%) & 81 & 81 & 0.975 \\
Diuretics (\%) & $135( \pm 23)$ & $133( \pm 21)$ & 0.429 \\
Systolic BP (mean) & $86( \pm 17)$ & $81( \pm 12)$ & 0.011 \\
Diastolic BP (mean) & 87 \\
\hline
\end{tabular}

In our study population, about $70 \%$ (149) of patients were taking beta-blockers with 93 controlled vs 56 with suboptimal control of heart rate. However, few patients were taking recommended or optimal dosage $\left(\begin{array}{llll}06 & \text { vs } & 02\end{array}\right.$ respectively). There was no significant association between beta-blocker dosage, concomitant therapies, and sub-optimal heart rate control.

\section{Discussion}

This study aimed to evaluate heart rate control in patients with stable chronic heart failure in three reference centers of heart failure management in Cameroon. We found that more than sixty-five percent of patients had suboptimal heart rate control.

In this study $64.8 \%$ of patients followed for stable chronic heart failure had poor heart rate control. These results are similar to those of Pastror-pérez, [10] but superior to those of Moran and Pourdjabbar, [11, 12] who found that $1 / 3$ of the population with chronic HF had poor HR control. This difference can be related to the high proportion of patients not taking a beta-blocker (30\%) in our study, unlike the other two, where this proportion was respectively $10.56 \%$ and $5.29 \%$. As the benefits of reducing $\mathrm{HR}$ in $\mathrm{CHF}$ is proven, identifying such a large population of uncontrolled patients had a significant clinical consequence. Indeed, given that a $1 \mathrm{bpm}$ drop in HR produces a $3 \%$ reduction in cardiac events, [9] increased follow-up and appropriate management can reduce morbimortality in this subgroup of the heart failure population.

Unachievement of recommended target heart rates were unrelated to age, gender, cardiovascular risk factors, and medication. These results corroborate those of Moran in Ireland [11], but they are contradictory to those described by studies reported in the literature [9]. In these studies, patients with $\mathrm{HR} \geq 70 \mathrm{bpm}$ were younger, had a lower ejection fraction, higher systolic blood pressure, and a more advanced NYHA stage than patients with FC $<70 \mathrm{bpm}$. This difference could be related to the fact that in our study population there were few young subjects, few severe alterations LVEF and stage 3 NYHA were poorly represented. There was no difference between patients receiving beta-blockers or not, but $67 \%$ of patients with $\mathrm{HR} \geq 70 \mathrm{bpm}$ were on beta-blocking agents, this can be explained by insufficient titration of betablockers, but we found no significant association between beta-blocker dose and poor HR control, results similar to those found in several studies [9, 11-13] thus evoking, on one hand, the theory of variability of responses to beta-blockers. Indeed, some patients may be poor responders to betablockers regardless of the dose [13]. On the other hand, may suggest that the bradycardic effect of beta-blockers alone is not always sufficient for optimal heart rate control. In this context, the use of more than one drug like recommended to achieve HR goals may be beneficial for patients with stable chronic HF and high HR [6]. The low prescription of ivabradine (1.69\% of patients) in our study can be explained by the cost of the drug or it could be a problem of indication. Indeed, according to the recommendations of the European Society of Cardiology, ivabradine is indicated in patients with stable chronic heart failure with a left ventricular ejection fraction $<35 \%$ and an $\mathrm{HR}>70 \mathrm{bpm}$ in sinus rhythm despite standard therapy including beta-blockers, optimaldose SRAA inhibitors or in case of intolerance or contraindications to beta-blockers, [6] but in our study population few had all these characteristics.

Limitations: use of the radial pulse palpation which is not the gold standard to measure heart rate. Indeed, the ECG Holter is the reference method for this evaluation but PastorPerez et al highlighted a strong concordance between the palpatory method and the Holter ECG nevertheless this concordance remains suboptimal for some lower values of heart rate. Our study was performed in reference management centers and this could be underestimated patient with poor heart rate control in our context since most of the patients are followed by a specialist and could therefore present better condition than others patients followed in primary care centers where there is no specialist and guidelines are not always respected due to insufficient technical plateau or unavailability of some molecules. Given that, poor heart rate control in our context might be more frequent than the proportion found in this study.

\section{Conclusion}

This study showed that more than a half of heart failure patients had sub-optimal heart rate control and stress the need to pay greater attention to heart rate control in patients with stable chronic heart failure in order to meet recommended targets that could improve patients' outcome in terms of life expectancy, hospitalizations, and quality of life.

\section{List of Abbreviations}

HR: Heart Rate; CHF: Chronic Heart Failure; LVEF: Left Ventricular Ejection Fraction; NYHA: New York Health Association.

\section{Funding}

The study received no funding. 


\section{Authors' Contributions}

Study conception and design: Audrey Joyce Foka, Kingue Samuel, Jérôme Boombhi, Ba Hamadou.

Data collection: Audrey Joyce Foka, Ahmadou M. Jingi, Sylvie Ndongo Amougou, Chris Nadege Nganou, Liliane Mfeukeu Kuate, Jérôme Boombhi, Ba Hamadou.

Statistical analysis: Audrey Joyce Foka, Ahmadou M. Jingi, Aurel T. Tankeu, Ba Hamadou.

Drafting: Kingue Samuel, Alain Menanga, Ba Hamadou, Jérôme Boombhi, Aurel T. Tankeu, Chris Nadège Nganou, Sylvie Ndongo Amougou, Liliane Kuate Mfeukeu, Chris Nadège Nganou.

Critical discussion and manuscript revision: Kingue Samuel, Ba Hamadou, Jérôme Boombhi, Aurel T. Tankeu, Audrey Joyce Foka, Ahmadou M. Jingi.

All the authors approved the final version of the manuscript.

\section{Competing Interests}

The authors declare that they have no competing interests.

\section{Acknowledgements}

We gratefully acknowledge all the participants who accepted to take part in this study.

\section{References}

[1] Olivia F, Sormani P, Contri R, Campana C, Carubelli V, Ciro $\mathrm{A}$ et al. Heart rate as a prognostic marker and therapeutic target in acute and chronic heart failure. Int J Cardiol. 2018; 253: 97-104.

[2] Dobre D, Borer JS, Fox K, Swedberg K, Adams KF, Cleland JG et al. Heart rate: a prognostic factor and therapeutic target in chronic heart failure the distinct roles of drugs with heart rate lowering properties. Eur J Heart Fail. 2014; 16 (1): 76-85.

[3] Böhm M, Swedberg K, Komajda M, Borer JS, Ford I, DubostBrama $\mathrm{A}$, et al. Heart rate as a risk factor in chronic heart failure (SHIFT): the association between heart rate and outcomes in a randomized placebo-controlled trial. Lancet Lond Engl. 2010; 376 (9744): 886-94.
[4] Fox K, Ford I, Steg PG, Tendera M, Robertson M, Ferrari R. Heart rate as a prognostic risk factor in patients with coronary artery disease and left-ventricular systolic dysfunction (BEAUTIFUL): a subgroup analysis of a randomized controlled trial. The Lancet. 2008; 372 (9641): 817-21.

[5] Ho JE, Bittner V, Demicco DA, Breazna A, Deedwania PC, Waters DD. Usefulness of heart rate at rest as a predictor of mortality, hospitalization for heart failure, myocardial infarction, and stroke in patients with stable coronary heart disease (Data from the Treating to New Targets [TNT] trial). Am J Cardiol. 2010; 105 (7): 905-11.

[6] Ponikowski P, Voors AA, Anker SD, Bueno H, Cleland JGF, Coats AJS, et al. 2016 ESC Guidelines for the diagnosis and treatment of acute and chronic heart failure. Eur Heart J. 2015; ehw128.

[7] Fowler MB, Vera-Llonch M, Oster G, Bristow MR, Cohn JN, Colucci WS, et al. Influence of carvedilol on hospitalizations in heart failure: incidence, resource utilization, and costs. J Am Coll Cardiol. 2001; 37 (6): 1692-9.

[8] Packer M, Bristow MR, Cohn JN, Colucci WS, Fowler MB, Gilbert EM, et al. The Effect of Carvedilol on Morbidity and Mortality in Patients with Chronic Heart Failure. N Engl J Med. 1996; 334 (21): 1349-55.

[9] Borer JS, Böhm M, Ford I, Komajda M, Tavazzi L, Sendon $\mathrm{JL}$, et al. Effect of ivabradine on recurrent hospitalization for worsening heart failure in patients with chronic systolic heart failure: the SHIFT Study. Eur Heart J. 2012; 33 (22): 2813-20.

[10] Pastor-Pérez FJ, Manzano-Fernández S, Goya-Esteban R, Pascual-Figal DA, Bravo IPG, Barquero-Pérez O, et al. Heart rate control in chronic heart failure: Resting versus mean heart rate with prolonged ambulatory ECG recording. Int J Cardiol. 2013; 170 (2): 45-7.

[11] Moran D, Buckley A, Daly K, Meaney B, Curtin R, O’Neill $\mathrm{JO}$, et al. Heart rate awareness in patients with chronic stable heart failure. A multi-center observational study. Int J Cardiol. 15 déc 2014; 177 (2): 380-4.

[12] Haddad R, Pourdjabbar A, Dwivedi G, Saikali A, Mielniczuk L, Stadnick E, et al. Heart rate control in patients with heart failure and left ventricular systolic dysfunction, a single center experience. Can J Cardiol. 2014; 30 (10): S197.

[13] McAlister FA, Wiebe N, Ezekowitz JA, Leung AA, Armstrong PW. Meta-analysis: beta-blocker dose, heart rate reduction, and death in patients with heart failure. Ann Intern Med. 2009; 150 (11): 784-94. 